\title{
Amiloidosis cardíaca en un paciente con mieloma múltiple
}

\section{(Cardiac amyloidosis in a patient with multiple myeloma)}

Ignacio Fallas-Mora, ${ }^{1}$ Eduardo Alvarado-Sánchez, ${ }^{1}$ Carla Brenes-Arguedas ${ }^{2}$

\section{Resumen}

La amiloidosis sistémica constituye una enfermedad poco frecuente, donde la infiltración cardíaca es la principal causa de morbimortalidad, sin importar la causa subyacente del depósito amiloide. Se reporta el caso de una paciente femenina de 48 años con síndrome nefrótico, insuficiencia cardíaca e inmunocompromiso, estableciéndose el diagnóstico de amiloidosis primaria con infiltración cardíaca secundaria a mieloma múltiple. Se discute brevemente la enfermedad, la importancia del juicio clínico apoyado en medios diagnósticos y los retos terapéuticos actuales.

Descriptores: amiloidosis cardíaca, amiloidosis primaria, miocardiopatía infiltrativa, insuficiencia cardíaca.

\section{Abstract}

Systemic amyloidosis constitutes a non common disease in which cardiac involvement is the leading cause of morbidity and mortality, regardless of the underlying pathogenesis of amyloid production. We present the case of a 48 years old female with nephrotic syndrome, heart failure and immunocompromise in which Primary Amyloidosis with cardiac involvement secondary to Multiple Myeloma is established as diagnosis. The disease is briefly discussed, as well as the value of clinical judgment supported on diagnostic means and the therapeutic challenges now days.

Keywords: Cardiac Amyloidosis, Primary Amyloidosis, Infiltrative Cardiomyopathy, Heart Failure.

Trabajo realizado en: Servicio de Cardiología, Hospital San Vicente de Paúl, Caja Costarricense de Seguro Social, Heredia, Costa Rica.

Afiliación de los autores: 'Servicio de Cardiología del Hospital San Vicente de Paúl, Heredia, Costa Rica. 'Área de Salud de San Rafael de Heredia, Costa Rica.

Abreviatura: amiloidosis primaria, AL.

Conflicto de interés: no existen conflictos de interés por declarar ni financiamiento externo para la preparación de este artículo.

凶drifallasm@gmail.com

ISSN 0001-6012/2019/61/3/131-133

Acta Médica Costarricense, () 2019

Colegio de Médicos y Cirujanos

de Costa Ricaa
Las miocardiopatías se definen como: "un grupo heterogéneo de enfermedades del miocardio relacionadas con disfunción mecánica, eléctrica, o ambas, que por lo general (pero no invariablemente) muestran hipertrofia o dilatación ventriculares inapropiadas y se deben a diversas causas, que a menudo son genéticas."

La amiloidosis cardíaca corresponde a una miocardiopatía infiltrativa-restrictiva que en ciertas formas puede asociarse con un componente tóxico. El término amiloide designa un trastorno poco común en el plegamiento de las proteínas con el depósito subsecuente de fibrillas insolubles en el espacio extracelular. Las enfermedades amiloides son definidas bioquímicamente por el precursor proteico que compone los depósitos de fibrillas, y a pesar de ser una enfermedad sistémica con infiltración multiorgánica con más de 30 proteínas identificadas como precursoras, solo algunas causan un depósito significativo en el tejido cardíaco. ${ }^{2}$ A continuación, se presenta un caso de infiltración amiloide cardíaca secundaria a mieloma múltiple (amiloidosis primaria), que permite resaltar las principales características de esta entidad.

\section{Presentación del caso}

Paciente femenina de 48 años con antecedentes patológicos personales de hipertensión arterial, hipotiroidismo secundario a tiroiditis de Hashimoto y colitis linfocítica, bajo medicación con enalapril $20 \mathrm{mg}$ vo Bid y levotiroxina $125 \mathrm{ug}$ vo al día. Presenta un episodio de shock séptico secundario a bronconeumonía adquirida en la comunidad meses antes de la valoración por Cardiología. Niega antecedentes patológicos familiares,

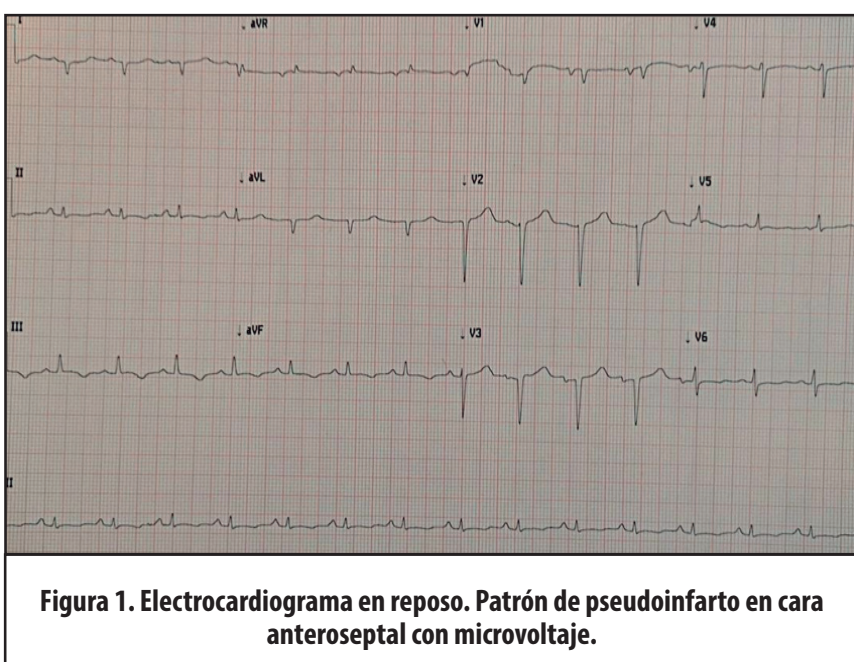


quirúrgicos y traumáticos. Se valora en el servicio de Cardiología por solicitud del nefrólogo asistencial, por presentar insuficiencia cardíaca en el transcurso de un síndrome nefrótico. En la anamnesis, la paciente únicamente manifiesta palpitaciones esporádicas y molestias inespecíficas en ambos pies. Al examen físico, se documenta ingurgitación yugular, hepatomegalia de $7 \mathrm{~cm}$ debajo del reborde costal derecho, de bordes lisos, redondeado, discretamente sensible a la palpación, así como esplenomegalia grado I y edemas bipodálicos. Se constatan cifras de tensión arterial con sistólica menor a 100 mmHG (dato prevalente en consultas previas desde el inicio de los síntomas).

Los estudios por enfermedades autoinmunes son negativos. Se cuantifica proteinuria $5 \mathrm{~g} / 24$ horas. La electroforesis de proteínas arroja una franca gammapatía monoclonal (inmunoglobulina G-2543mg/dL, inmunoglobulina A-105mg/ dL, inmunoglobulina M- $66 \mathrm{mg} / \mathrm{dL}$, cadenas kappa- $104 \mathrm{mg} / \mathrm{dL}$, cadenas lambda- $616 \mathrm{mg} / \mathrm{dL}$ ). Ante la sospecha de un mieloma múltiple con amiloidosis sistémica asociada, se solicitan biopsias y estudios inmunohistoquímicos.

La biopsia renal muestra aumento difuso de matriz mesangial por material denso que tiñe positivamente con cristal violeta y rojo congo, así como atrofia focal leve de túbulos y fibrosis focal leve de intersticio. En el estudio inmunohistoquímico del fragmento obtenido de la médula ósea, se observa una infiltración intersticial por células plasmáticas con fenotipo anómalo que compromete el $10 \%$ de los elementos hematopoyéticos. De igual forma, se visualiza depósito amiloide birrefringente con luz polarizada con la tinción de rojo congo.

El electrocardiograma muestra baja amplitud de voltajes y patrón de pseudoinfarto en cara anterior (figura 1). Se realiza holter de 24 horas que no registra alteraciones significativas.
El ecocardiograma transtorácico (figura 2) revela hipertrofia leve del ventrículo izquierdo con remodelado severo concéntrico e hipertrofia moderada del ventrículo derecho, con función contráctil biventricular conservada. El strain 2D longitudinal global se encuentra deprimido en segmentos basales y medios, pero conservado en segmento apicales. Las paredes de ambos ventrículos lucen engrosadas de aspecto infiltrado. El atrio izquierdo luce dilatado moderadamente. Asocia disfunción diastólica tipo III con patrón restrictivo e insuficiencia tricuspídea severa por movimiento restrictivo de las valvas (patrón III B).

El tratamiento de la insuficiencia cardíaca se ha basado en la titulación de diuréticos y suspensión del enalapril. Por parte del Departamento de Hematología, se inició régimen de bortezomid. Actualmente, la paciente se encuentra en control ambulatorio con estabilidad de su enfermedad.

\section{Discusión}

Las enfermedades poco frecuentes constituyen un reto diagnóstico en la práctica clínica, pues, de pasar inadvertidas, repercuten negativamente en el pronóstico y la calidad de vida de los pacientes. Se registró una incidencia de amiloidosis sistémica de al menos 4/1 000 000,00 habitantes en la población inglesa de 2008, según datos extrapolados de registros epidemiológicos. ${ }^{3}$ La amiloidosis primaria (AL) aparece como consecuencia de trastornos proliferativos de células plasmáticas clonales en las que inmunoglobulinas de cadena ligera mal plegadas se depositan como fibrillas amiloides en múltiples órganos, incluyendo el corazón en cerca de la mitad

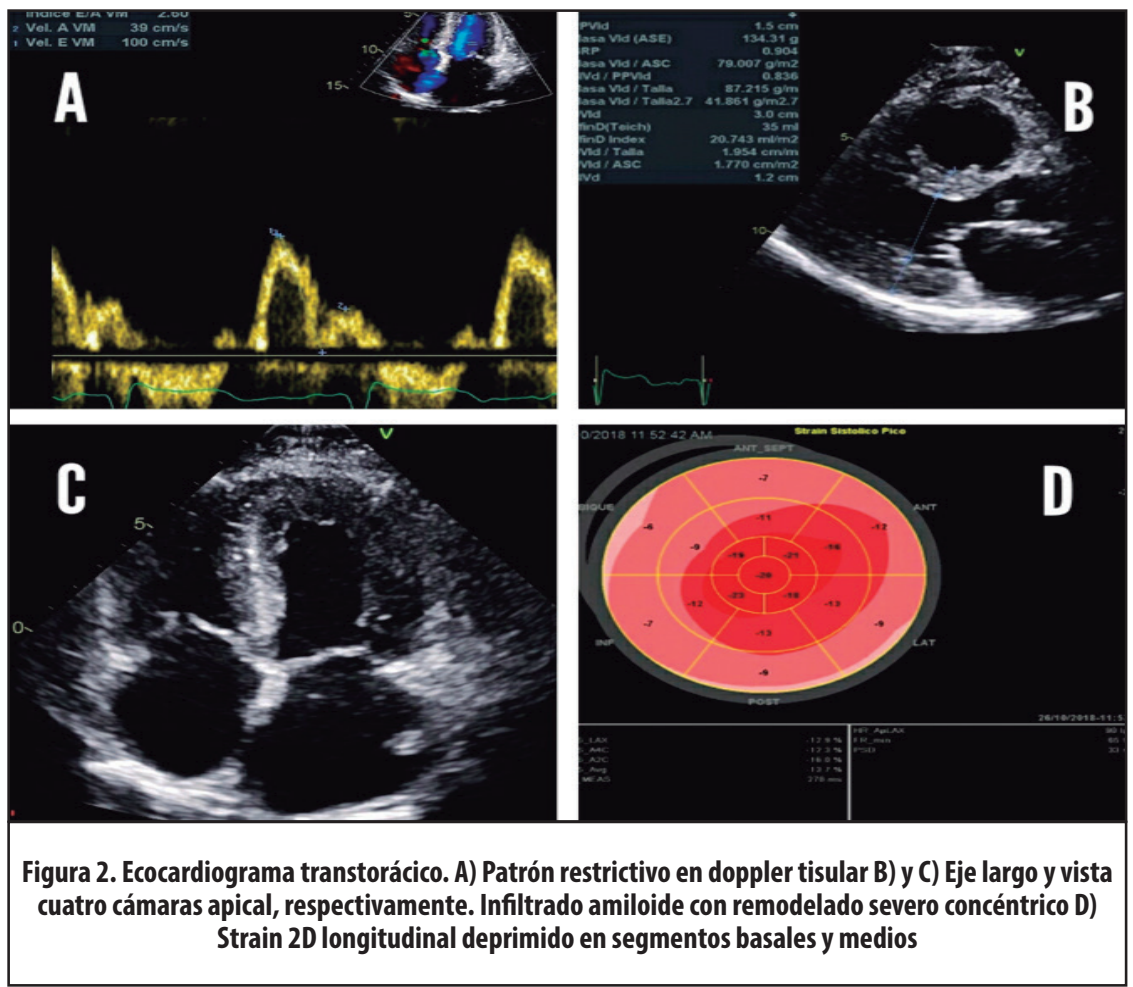




\section{Paciente con infiltración cardíaca amiloide / Fallas-Mora et al}

de los casos. ${ }^{4}$ Precisamente, el caso clínico presentado se trata de una paciente con AL secundaria a mieloma múltiple, cuyo diagnóstico clínico se basó en las manifestaciones sistémicas de la enfermedad e inmunohistológicamente fue confirmado por las biopsias, en las cuales la tinción positiva de rojo congo establece la presencia de proteínas amiloides, y el infiltrado intersticial de células plasmáticas con fenotipo anómalo que compromete el $10 \%$ de los elementos hematopoyéticos en la muestra de médula ósea, advierte el diagnóstico de mieloma múltiple.

Llama la atención que el curso de la enfermedad permite apreciar datos semiológicos, electrocardiográficos y de imagen, muy enriquecedores. Clínicamente, el edema bipodálico obligaba en un inicio al diagnóstico diferencial de entidades causantes de descompensación hidrópica. Sin embargo, luego se establece una semiogenia multicausal (hipoproteinemia por síndrome nefrótico y congestión venosa por insuficiencia cardíaca derecha). Las características descritas a la palpación de la hepatomegalia, son sugestivas de congestión hepática, y asociadas a ingurgitación yugular, no se justificaban, sino por insuficiencia cardíaca derecha asociada. Además, se aprecia una "normalización" de la hipertensión arterial con necesidad de suspender el inhibidor de la enzima convertidora de angiotensina utilizada como tratamiento crónico. El ECG muestra patrón OS en cara anteroseptal, que obliga al diagnóstico diferencial con cardiopatía isquémica, sin embargo, la asociación con microvoltajes y la ausencia de trastornos segmentarios en la motilidad de la pared ventricular en el ecocardiograma, descartan esta entidad. El ecocardiograma requiere plantear diferencias con entidades como la miocardiopatía hipertrófica, que si bien justifica el aumento en el grosor de la pared ventricular, no tiende a infiltrar en forma difusa al ventrículo y respeta el septo interatrial. Los estudios de inmunohistoquímica ya citados permitieron no solo establecer una relación etiológica a las manifestaciones renales, cardiovasculares e inmunológicas de la paciente, sino también identificar el precursor proteico específico para el depósito amiloide, y con ello, se definió el tratamiento. En AL, el régimen con bortezomib ha demostrado beneficios en el control de la discrasia de células plasmáticas y aumento en las tasas de superviviencia. ${ }^{4}$ El trasplante hepático únicamente es razonable en amiloidosis cardíaca por transtirretina en su variante genética, y por lo tanto, no es planteable en esta paciente. ${ }^{5}$ Cabe resaltar que para el manejo actual de la insuficiencia cardíaca en este contexto, solo se dispone del uso de diuréticos. Si se toma en cuenta que la infiltración cardíaca en la amiloidosis sistémica corresponde a la principal causa de morb-mortalidad en pacientes con esta entidad, con independencia de la patogénesis involucrada en la producción amiloide, ${ }^{6}$ es menester la investigación de rutas fisiopatológicas, con el objetivo de encontrar dianas terapéuticas que permitan modificar la evolución natural de la afección cardíaca por amiloidosis.

La amiloidosis cardíaca constituye una miocardiopatía infiltrativa poco frecuente, la cual se solapa con características restrictivas del miocardio. El diagnóstico requiere un juicio clínico perspicaz y su identificación inmunohistoquímica. Se requiere nuevos avances terapéuticos para mejorar el pronóstico y la calidad de vida de los pacientes.

\section{Referencias}

1. Maron BJ, Towbin JA, Thiene G, Antzelevith C, Corrado D, Arnett D, et al. Contemporary definitions and classification of the cardiomyopathies: an American Heart Association scientific statement from the Council on Clinical Cardiology, Heart Failure and Transplantation Committee; Quality of Care and Outcomes Research and Functional Genomics and Translational Biology Interdisciplinary Working Group; and Council on Epidemiology and Prevention. Circulation. 2006; 113:1807-1816.

2. García-Pavía P, Tomé-Esteban MT, Rapezzi C. [Amiloidosis, also an illness of the heart]. Rev Esp Cardio. 2011;64:797-808. Spanish.

3. Pinney JH, Smith CJ, Taube JB, Lachmann HJ, Venner CP, Gibbs SD, et al. Systemic amyloidosis in England: an epidemiological study. Br J Haematol. 2013;161(4):525-532.

4. Martínez- Naharro A, Hawkins PN, Fontana M. Cardiac amyloidosis. Clin Med. 2018;18,2:S30-S5.

5. González López E, López Sainz, García Pavia P. Diagnosis and treatment of transthyretin

6. amyloidosis. progress and hope. Rev Esp Cardio. 2017;70(11):991-1004. Spanish. 